\title{
Dopamine Enhances Item Novelty Detection via Hippocampal and Associative Recall via Left Lateral Prefrontal Cortex Mechanisms
}

\author{
@Mareike Clos, ${ }^{1}$ @Nico Bunzeck, ${ }^{1,2}$ and $\oplus^{-T o b i a s ~ S o m m e r ~}{ }^{1}$ \\ ${ }^{1}$ Department of Systems Neuroscience, University Medical Center Hamburg-Eppendorf, 20246 Hamburg, Germany, and 2Institute of Psychology I, \\ University of Lübeck, 23562 Lübeck, Germany
}

The involvement of fronto-striatal circuits in item and associative memory retrieval as well as in the stabilization of memories by retrieval practice suggests that both retrieval and re-encoding of stored memories might rely on dopaminergic mechanisms in humans. We tested these hypotheses in a placebo-controlled pharmacological fMRI study using $2 \mathrm{mg}$ of the D2 antagonist haloperidol administered acutely before a cued associative recall task of previously encoded picture-word pairs in 53 healthy humans of both sexes. The cued associative recall was moreover repeated $3 \mathrm{~d}$ later outside the scanner without pharmacological intervention. Dopaminergic modulation significantly improved associative recall performance and recognition accuracy of verbal items. Moreover, we observed a significant dopamine effect on re-encoding in terms of increased specificity of associative memories from the first to the second cued associative recall. Better association memory under haloperidol was linked with higher activity in the left lateral prefrontal cortex and right parietal cortex, suggesting that dopamine facilitates associative retrieval through increased recruitment of frontoparietal monitoring processes. In contrast, improved recognition of verbal items under haloperidol was reflected by enhanced novelty detection in the hippocampus and increased activity in saliency networks. Together, these results show distinct but concomitant positive effects of dopamine on associative recall and item recognition and suggest that the specificity of associative recall through re-encoding mechanisms is likewise augmented by dopamine.

Key words: associative memory; episodic memory; fMRI; haloperidol; memory retrieval; re-encoding

Significance Statement

Although the neurotransmitter dopamine has been linked with learning and memory for a long time, dopaminergic effects on item recognition in humans were demonstrated only recently. The involvement of fronto-striatal monitoring processes in association retrieval suggests that associative memory might be particularly affected by dopamine. Moreover, fronto-striatal dopaminergic signals have been hypothesized to determine the updating and re-encoding of previously retrieved memories. We here demonstrate clear facilitative effects of dopamine on associative recall and item recognition mediated by prefrontal and hippocampal mechanisms respectively. Additionally, effects on re-encoding were reflected by increased specificity of associative memories. These results augment our understanding of dopaminergic processes in episodic memory retrieval and offer new perspectives on memory impairments in dopamine-related disorders and their treatment.

\section{Introduction}

The retrieval of stored associative memory representations from the medial temporal lobe (MTL) is critically shaped by top-down

\footnotetext{
Received March 1, 2019; revised July 4, 2019; accepted July 16, 2019.

Author contributions: M.C. and T.S. designed research; M.C. performed research; N.B. and T.S. contributed unpublished reagents/analytic tools; M.C. analyzed data; M.C., N.B., and T.S. wrote the paper.

This work was supported by a Grant from the Deutsche Forschungsgemeinschaft to T.S. and N.B. (DFG SO 952/

3-1). The funders had no role in study design, data collection and analysis, decision to publish, or preparation of the paper. We thank the University of Minnesota Center for Magnetic Resonance Research for providing the image reconstruction algorithm for the simultaneous multi-slice acquisitions.

The authors declare no competing financial interests.
}

inputs from prefrontal cortex (PFC) in monkeys (Hasegawa et al., 1998; Tomita et al., 1999; Miyashita and Hayashi, 2000). Consistent with these findings, human fMRI studies have linked association retrieval with prefrontal and striatal activations (Spaniol et al., 2009; Kim, 2013). The contribution of the lateral PFC to association memory is thought to reflect increased prefrontal cognitive control and monitoring demands that accompany this 
retrieval of learned associations (Simons and Spiers, 2003; Dobbins and Han, 2006; Badre and Wagner, 2007; Kim, 2013). Cognitive control processes involve dopaminergic stimulation of the PFC (Miller, 2000; Puig et al., 2014) and dopamine-dependent fronto-striatal interactions (Cools, 2016). This suggests that dopaminergic mechanisms could also play a role in the retrieval of associative memories.

Indeed, there is some evidence linking aberrant dopamine function and performance in source memory tasks where contextual details need to be retrieved (Montoya et al., 2008; Drag et al., 2009; Li et al., 2010). However, these studies did not constrain dopaminergic modulation to the retrieval phase specifically and therefore cannot distinguish between dopaminergic effects on encoding and on retrieval of associations. For item memory, in contrast, there is evidence for retrieval-specific dopaminergic effects on recognition facilitation in animals (Sara, 1986; Chugh et al., 1991) and in humans (Clos et al., 2019). In particular, we recently demonstrated that dopaminergic modulation applied post-learning improves midbrain-hippocampal memory retrieval of previously encoded picture items (Clos et al., 2019). In the current study, we investigated whether this dopaminergic effect is also present for the retrieval of previously learned associations and how the dopaminergic modulation affects prefrontal mechanisms underlying association retrieval.

Additionally, the consistent observation of striatal and prefrontal activity during memory retrieval (Han et al., 2010; Kim, 2013; Schwarze et al., 2013; Clos et al., 2015) has been proposed to reflect fronto-striatal dopaminergic processes signaling the importance and behavioral relevance of retrieved information (Scimeca and Badre, 2012). Similar to dopaminergic effects on encoding (Lisman and Grace, 2005), dopamine might thus affect the updating and re-encoding of previously retrieved memories based on current goals and presumed future utility (Scimeca and Badre, 2012). In agreement with this, the stabilization of memory representations after repeated testing compared with re-study has been shown to recruit fronto-striatal and midbrain regions (van den Broek et al., 2013; Liu et al., 2014; Wiklund-Hörnqvist et al., 2017). This improvement of memory performance by retrieval practice is known as the "testing effect" and has been observed also in the absence of accuracy feedback (Roediger and Butler, 2011; Rowland, 2014).

To disentangle the potential dopaminergic effects on item memory and associative memory retrieval and re-encoding, we pharmacologically modulated dopaminergic neurotransmission during a cued association recall task of previously learned picture-word pairs while measuring fMRI activity. Memory was additionally tested 3 d later outside of the scanner. This study was part of a series of randomized, placebo-controlled pharmacological experiments (Clos et al., 2018, 2019) in which dopamine level was manipulated by $2 \mathrm{mg}$ of the D2-antagonist haloperidol. Importantly, such acute low doses of D2-antagonists are thought to preferably block presynaptic D2-autoreceptors (Frank and O'Reilly, 2006; Knutson and Gibbs, 2007; Clos et al., 2018, 2019), which regulate dopamine release (Ford, 2014). Therefore, their blockade potentiates phasic dopamine release (Dugast et al., 1997; Pehek, 1999; Jaworski et al., 2001; Garris et al., 2003; Chen et al., 2005). As dopamine release in the striatum is paralleled by increased striatal cerebral blood volume and BOLD MRI signals (Chen et al., 1997; Choi et al., 2006), D2-antagonist-induced amplification of the presumably phasic dopamine release evoked by cued association recall should be reflected by increased BOLD activity in dopaminergic regions involved in memory retrieval (Clos et al., 2019). As haloperidol was administered only after encoding, we could examine the effects of dopaminergic modulation specifically on retrieval processes.

\section{Materials and Methods}

\section{Participants}

We used a double-blind, placebo-controlled between-group design with 54 participants in total as part of a larger study (Clos et al., 2018, 2019). We had to use a between-group design rather than a more powerful within-group crossover design because of the limited stimulus set and possible interference effects between the picture-word pairs. Additionally, also another task measured in this sample (Clos et al., 2018) was incompatible with a within-group design. Fifty-three of these participants ( 13 males, mean age $24 \pm 2.9$ years; 27 haloperidol and 26 placebo) took part in the cued association recall paradigm. The study was approved by the local ethics committee of the Hamburg medical association and written consent was given by each participant before the start of the study. Additionally, all participants were screened by a physician for previous or current physical or mental diseases, medication, or drug use. The participants were informed about the study and about the potential risks and side effects of haloperidol. Moreover, the participants were instructed to restrain from caffeine, nicotine, and alcohol on the day of the fMRI testing. All participants were monitored for side effects and mood effects after drug administration and had to indicate the substance (haloperidol or placebo) they thought they had received at the end of the study. Additionally, working memory span was measured at baseline and again after drug administration following the fMRI scanning using a complex span task (Unsworth et al., 2009) and a digit and block span task (Kessels et al., 2008; Clos et al., 2019).

\section{Stimuli}

160 picture-word pairs were used during the encoding phase. The pictures were photographs of 40 male and 40 female famous faces as well as 40 natural and 40 man-made famous scenes. The 160 target words shown during encoding as well as the 120 words used as lures in the memory tests were concrete German nouns with 3-11 letters selected from the Leipzig Corpora Collection (Goldhahn et al., 2012). Target and lure words were matched for word frequency and word length.

\section{Experimental design}

Behavioral encoding session. Encoding for the fMRI experiment took place outside the scanner before drug administration (Fig. 1A). In each of the 160 encoding trials the word-picture pairs were presented for $4 \mathrm{~s}$. Note that we showed each pair just once, as we aimed at one-shot learning to be able to investigate purely episodic memory. The target word was shown above the picture. Below the picture the name of the person or the scene was provided. The participants were instructed to memorize the target word together with the associated picture by visually imagining the depicted face or scene together with the object described by the word. Each picture-word pair was followed by an active baseline condition (ISI $3 \mathrm{~s}$, arrow pointing task) in which the direction of arrows presented for $500 \mathrm{~ms}$ had to be indicated by button press.

Association retrieval session under haloperidol/placebo in the fMRI scanner. After the encoding session, the participants received a tablet containing $2 \mathrm{mg}$ of haloperidol or placebo. Two hours and $30 \mathrm{~min}$ after tablet ingestion the participants first performed an unrelated picture recognition task of unfamiliar street scenes in the fMRI scanner described by Clos et al. (2019). Note that these picture stimuli were very different from the famous place and face pictures used for word-picture association encoding and therefore unlikely lead to interference between tasks. Approximately $50 \mathrm{~min}$ later, the participants started the cued association recall inside the scanner. During this task, all 160 previously shown words were presented randomly intermixed with 120 new words (lures). For each word (presented for $4 \mathrm{~s}$, ISI 2-4 s), the participants had to press one of six buttons corresponding to the response options "new word not shown during encoding", "word remembered but not the associated picture", "some kind of scene remembered", "specific scene remembered", "some kind of face remembered", "specific face remembered". That is, the participants used a combined rating scale to indicate simultaneously 
item memory (old or new word) and the remembered association category (face or scene) if applicable (Fig. 1B). Feedback was not provided. The completion of this task took $\sim 40$ min.

Behavioral association retrieval session. Three days later, the participants returned to the lab and repeated the cued association recall to examine effects of dopamine on re-encoding during the first retrieval session. In this selfpaced cued association recall task performed on the computer all 160 old words (shown in the encoding session together with a picture and tested in the first retrieval session) and the 120 lure words from the first retrieval session were presented in random order. That is, we used the same set of word stimuli as in the first retrieval session because we were mostly interested in re-encoding effects on associative rather than item memory because stronger effects of repeated testing were previously reported for associative material (Rowland, 2014). Additionally, the amount of suitable concrete and frequent noun stimuli was too low to create another full list of 120 lure words for the second retrieval session. Participants were explicitly instructed to respond exclusively to word items shown during initial encoding as old and treat word items only remembered from the first retrieval session as new. For each word, a choice of 12 response options specifying the remembered picture category and its specificity was presented until the participants indicated their response by mouse click on one of the categories (new word not shown during encoding, word remembered but not the associated picture, "scene", "natural scene", "man-made scene", "specific natural scene", "specific man-made scene", "face", "male face", "female face", "specific male face", "specific female face"; Fig. 1C).

$f M R I$ data acquisition and preprocessing. One placebo dataset was acquired only behaviorally because of scanner problems. For the remaining 52 participants, functional MR images were obtained during the first cued association recall test on a $3 \mathrm{~T}$ system Siemens Trio using single-shot echo-planar imaging with parallel imaging]GRAPPA (Griswold et al., 2002), in-plane acceleration factor 2] and simultaneous multi-slice acquisitions (Feinberg et al., 2010; Moeller et al., 2010; Setsompop et al., 2012; Xu et al., 2013; "multiband," slice acceleration factor $2 ; \mathrm{TR}=1.98 \mathrm{~s}, \mathrm{TE}=26 \mathrm{~ms}$, flip angle $=$ $70^{\circ}, 64$ axial slices, voxel size $2 \times 2 \times 2 \mathrm{~mm}^{3}$ ). The corresponding image reconstruction algorithm was provided by the University of Minnesota Center for Magnetic Resonance Research. In addition, an anatomical high-resolution T1-weighted image $(\mathrm{TR}=2.3 \mathrm{~s}$, TE $=2.98 \mathrm{~ms}$, flip angle $=9^{\circ}, 192$ sagittal slices, voxel size $\left.1 \times 1 \times 1 \mathrm{~mm}^{3}\right)$ and an anatomical magnetization transfer $(\mathrm{MT})$ image $(\mathrm{TR}=14 \mathrm{~ms}$, $\mathrm{TE}=3.2 \mathrm{~ms}$, flip angle $=6^{\circ}, 240$ coronal slices, voxel size $1 \times 1 \times 1 \mathrm{~mm}^{3}$ ) was acquired for each participant.

The data were preprocessed using SPM12 (RRID:SCR_007037). The first five EPI images were discarded to allow for magnetic-field saturation. EPI images corrected for motion and for the interaction between motion and distortion (using the unwarping procedure). Anatomical T1-weighted images were normalized to standard MNI space using DARTEL normalization. Subsequently, the EPI images and the MT image were coregistered with the normalized T1 image and the DARTEL normalization parameters were applied to the EPI images and the MT image. Finally, these normalized EPI images were spa- tially smoothed with a Gaussian kernel of $8 \mathrm{~mm}$ full-width at halfmaximum.

\section{Statistical analysis}

Behavioral data. All behavioral data were analyzed using SPSS 21.0.0 (SPSS; RRID:SCR_002865) and MATLAB R2013a (MathWorks; RRID:SCR_001622).

Item memory. For the analysis of item memory performance in the retrieval sessions, we pooled together all associative and old word responses as "old items" regardless of the accuracy of the association recall. Trials were sorted into the response categories item hits (correct oldresponses), item correct rejections (CR; correct new-responses), item false alarms (FA; incorrect old-responses), and item misses (incorrect new-responses). Item memory performance measured as discrimination sensitivity $\left(d^{\prime}\right)$ as well as response bias c were computed according to the signal detection theory framework (Macmillan and Creelman, 2004) and compared between groups using independent samples $t$ tests at Retrieval I.

Associative memory. Associative memory for item hits was evaluated by computing the corrected associative memory (CAM) score across all item hit trials with associative responses collapsed across specificity level. This overall CAM score was derived for each participant by taking the associative hit rate (i.e., proportion of face-responses to recognized 
Table 1. Overview of used fMRI models

\begin{tabular}{|c|c|c|c|}
\hline & 1st level regressors ${ }^{a}$ & Contrasts 2nd level: main effects & Contrasts 2nd level: group differences ${ }^{b}$ \\
\hline Model I & Word onsets, missing response trials & n.a. & $\mathrm{H}>\mathrm{P}$ \\
\hline \multirow[t]{2}{*}{ Model II } & \multirow[t]{2}{*}{$\begin{array}{l}\text { Associative hits, associative FAs, associative } \\
\text { misses, item CR, item FAs, item misses, } \\
\text { missing response trials }\end{array}$} & $\begin{array}{l}\text { Item retrieval success: [associative hits }+ \text { associative } \\
\text { FAs }+ \text { associative misses] }>\text { item } C R \\
\text { Item novelty detection: item CR }>\text { [associative hits }+ \text { as- } \\
\text { sociative FAs + associative misses] }\end{array}$ & $\begin{array}{l}\text { Group } \times \text { new/old: }[\text { haloperidol (item CRs }+ \text { item } \\
\text { FAs) }>\text { (associative hits }+ \text { associative FAs }+ \text { associa- } \\
\text { tive misses }+ \text { item misses) }]>[\text { placebo (item } \\
\text { (Rs }+ \text { item FAs) }>\text { (associative hits }+ \text { associative } \\
\text { FAs }+ \text { associative misses }+ \text { item misses) }]\end{array}$ \\
\hline & & $\begin{array}{l}\text { Association retrieval success: associative hits }>\text { associative } \\
\text { misses }\end{array}$ & $\begin{array}{l}\text { Group } \times \text { association recall success: haloperidol (associative } \\
\text { hits }>\text { associative misses) }>\text { placebo (associative } \\
\text { hits }>\text { associative misses) }\end{array}$ \\
\hline \multirow[t]{2}{*}{ Model III } & \multirow{2}{*}{$\begin{array}{l}\text { Associative face hits, associative face FAs, } \\
\text { associative scene hits, associative scene } \\
\text { FAs, item C } R \text {, item FAs, item misses, miss- } \\
\text { ing response trials }\end{array}$} & $\begin{array}{l}\text { Face reinstatement: associative face hits }>\text { associative } \\
\text { scene hits }\end{array}$ & $\begin{array}{l}\text { Group } \times \text { face reinstatement: haloperidol (associative face } \\
\text { hits }>\text { associative scene hits) }>\text { (placebo (associative } \\
\text { face hits }>\text { associative scene hits) }\end{array}$ \\
\hline & & $\begin{array}{l}\text { Scene reinstatement: associative scene hits }>\text { associative } \\
\text { face hits }\end{array}$ & $\begin{array}{l}\text { Group } \times \text { scene reinstatement: haloperidol (associative } \\
\text { scene hits }>\text { associative face hits) }>\text { (placebo (associa- } \\
\text { tive scene hits }>\text { associative face hits) }\end{array}$ \\
\hline
\end{tabular}

${ }^{a}$ Italics indicate regressors of non-interest not included in the corresponding second-level model.

${ }^{b}$ Group differences were always tested bidirectional.

words paired with face stimuli during encoding and scene-responses to recognized words paired with scene stimuli during encoding, respectively) and subtracting the associative FA rate (i.e., proportion of sceneresponses to recognized words paired with face stimuli during encoding and proportion of face-responses to recognized words paired with scene stimuli during encoding, respectively) from it. The overall CAM score was compared between groups using independent samples $t$ tests at Retrieval I and $2 \times 2$ repeated-measures ANOVAs with the factors group and time at Retrieval II. Additionally, we compared the relative frequencies of associative hits and associative FAs using a $2 \times 2 \times 2 \times 2$ repeatedmeasures ANOVA with the factors group, accuracy (associative hit/FA), specificity (general/specific associative response) and trial type (scene/ face) as well as the frequency of associative miss responses using a $2 \times 2$ repeated-measures ANOVA with the factors group and trial type (scene/ face) for each retrieval time point. Finally, we compared the frequency of general versus specific associative responses between groups for item FA trials to test for drug effects on the tendency to report associations using a $2 \times 2$ repeated-measures ANOVA with the factors group and specificity for each retrieval time point.

$R e$-encoding. Effects of re-encoding were evaluated by $2 \times 2$ repeatedmeasures ANOVAs with the factors group and time to test for changes in item recognition ( $d^{\prime}$ and bias $c$ ) and associative recall (overall CAM) from Retrieval I to Retrieval II. Additionally, because the strongest effects of repeated testing are observed for cued recall (rather than recognition; Rowland, 2014) and an increase in memory specificity has previously been reported for repeated testing in the associative domain (Sommer et al., 2008), we examined dopaminergic effects on re-encoding affecting associative memory specificity in more detail. To this end, we computed the CAM separately for general and specific associative responses on item hit trials for both retrieval time points (collapsing the categorical and subcategorical responses Retrieval II responses into a single specific response category) and compared them between groups using a $2 \times 2 \times 2$ repeated-measures ANOVA on the CAM score with the factors group, time (Retrieval I/II), and specificity (general/specific associative response. Moreover, we examined effects of re-encoding on the frequency of associative responses at Retrieval II. To this end, we conducted a $2 \times$ $2 \times 2 \times 2 \times 2$ repeated-measures ANOVA on the frequency of associative responses for item hits with the factors group, time (Retrieval I/II), accuracy (associative hit/FA), specificity (general/specific associative response; collapsing the categorical and subcategorical responses Retrieval II responses into one specific response category), and trial type (scene/ face). As an additional control analysis, we also compared the frequency of associative miss responses and the frequency of general and specific FA trials across retrieval time points using $2 \times 2 \times 2$ repeated-measures ANOVAs (with the factors group, time, and trial type for associative misses and group, time and specificity for item FAs, respectively). However, note that the testing effect is usually examined by comparing re- encoded (previously tested) stimuli with non-re-encoded (previously non-tested) stimuli. Unfortunately, such a direct comparison was not feasible within our design, as this would have required testing only half of the word items at Retrieval I and the other half only at Retrieval II. This approach would thus have severely decreased the amount of trials available for the fMRI analysis of Retrieval I, which was the main focus of the study. Doubling the amount of to-be-encoded word-picture pairs would neither have been a practicable alternative because it would have rendered the encoding even more challenging (the limited amount of suitable stimuli notwithstanding).

$f M R I$ data. Univariate single subject (first level) and group (second level) statistics were conducted using the general linear model as implemented in SPM12. Low-frequency signal drifts were removed by using a high-pass filter with a cutoff period of $128 \mathrm{~s}$. Note that we pooled together trials with general and specific association responses in all reported fMRI analyses, as the behavioral results did not reveal any group differences in specificity of associations at this first retrieval session (see Results section: Retrieval I Association recall). Moreover, specific association trials were too few for a sound fMRI analysis. This low number of specific association responses probably resulted from the combination of the time interval between encoding and testing (necessitated by the long time-topeak duration of haloperidol and the measurement of the preceding recognition memory task) and our focus on episodic memory, which precluded repeated presentation of to-be-learned association pairs during encoding. For trials with missing responses ( $\sim 3 \%$ of all trials in both groups), a nuisance regressor was included in all first-level models.

To test for general group differences due to haloperidol, we set up a first first-level model in which delta functions marking all word onset trials were convolved with the canonical hemodynamic response function to create an event-related regressor for word onset during retrieval (cf. Clos et al., 2019). The resulting $\beta$ representing word onsets was compared between groups using independent-samples $t$ tests on the second level (Table 1).

To examine effects of haloperidol on item and associative memory, we used a second first-level model where word onsets (collapsed across face and scene associations) were grouped into associative hit trials (item hits with correct association recall), associative FA trials (item hits with incorrect association recall), associative miss trials (item hits with forgotten associations), item CR trials, item FA trials (collapsed across all associative responses), and item miss trials. Note that the accuracy of the association recall was evaluated based on the selection of the correct association category (scene or face). The corresponding six betas (associative hits, associative FAs, associative misses, item CRs, item FAs, item misses) were fed into a second-level ANOVA. Main effects across groups of item retrieval success (item hits $>$ item CR) and item novelty detection (item CR $>$ item hits) were examined using linear contrasts summed across associative responses. Main effects across groups for association 
Table 2. Demographics and baseline performance

\begin{tabular}{|c|c|c|c|}
\hline & Placebo, $n=26$ & Haloperidol, $n=27$ & Statistics \\
\hline Age & $24.42 \pm 3.3$ years & $23.56 \pm 2.5$ years & $t_{(51)}=1.07, p=0.29$ \\
\hline Sex & 7 males, 19 females & 6 males, 21 females & $\chi_{(1)}^{2}=0.16, p=0.69$ \\
\hline Weight & $65.54 \pm 6.9 \mathrm{~kg}$ & $65.74 \pm 10.6 \mathrm{~kg}$ & $t_{(51)}=-0.08, p=0.94$ \\
\hline \multirow[t]{3}{*}{ Baseline WM: complex span/symmetry accuracy/RT symmetry rating } & $25.92 \pm 7.4$ & $27.85 \pm 7.0$ & $t_{(51)}=-0.98, p=0.33$ \\
\hline & $96.2 \pm 4.3 \%$ & $96.7 \pm 3.7 \%$ & $t_{(51)}=-0.46, p=0.64$ \\
\hline & $2.19 \pm 1.47 \mathrm{~s}$ & $1.67 \pm 0.63 \mathrm{~s}$ & $t_{(33.59)}=1.67, p=0.11$ \\
\hline \multirow[t]{2}{*}{ Baseline WM: digit span forward/backward } & $8.23 \pm 1.3$ & $8.30 \pm 1.7$ & $t_{(51)}=-1.16, p=0.87$ \\
\hline & $9.08 \pm 2.2$ & $8.67 \pm 1.7$ & $t_{(50)}=0.76, p=0.45$ \\
\hline \multirow[t]{2}{*}{ Baseline WM: block span forward/backward } & $9.88 \pm 1.7$ & $10.44 \pm 2.0$ & $t_{(50)}=-1.09, p=0.28$ \\
\hline & $9.12 \pm 1.9$ & $9.81 \pm 1.6$ & $t_{(50)}=-1.41, p=0.16$ \\
\hline Baseline WM: z-summary score & $-0.07 \pm 0.69$ & $0.08 \pm 0.60$ & $t_{(51)}=-0.87, p=0.39$ \\
\hline \multirow[t]{2}{*}{ Baseline encoding: categorization accuracy arrows/ RT } & $86 \pm 9.0 \%$ & $86 \pm 10.5 \%$ & $t_{(50)}=0.25, p=0.80$ \\
\hline & $0.38 \pm 0.01 \mathrm{~s}$ & $0.38 \pm 0.02 \mathrm{~s}$ & $t_{(50)}=0.32, p=0.98$ \\
\hline
\end{tabular}

\pm Denotes the standard variation.

retrieval success were examined using the linear contrast (associative hits $>$ associative misses). The analysis of fMRI group differences in item memory was guided by the behavioral results indicating that performance differences between groups were evident only for new items (see Results section: Retrieval I Item memory). Therefore, we compared the groups for fMRI activity in response to factual new relative to factual old items regardless of associative memory (group $\times$ new/old interaction: [haloperidol (item CRs + item FAs $)>($ item hits + item misses $)]>$ [placebo (item CR + item FA) $>$ (item hits + item misses)] and vice versa). Moreover, we compared the groups for fMRI activity in response to correct new trials relative to all other trials using a minimum statistic conjunction [(haloperidol item CRs $>$ item hits) $>$ (placebo item CR $>$ item hits) $]$ and [(haloperidol item $\mathrm{CR}>$ item FA) $>$ (placebo item $\mathrm{CR}>$ item FA)] and [(haloperidol item $\mathrm{CR}>$ item misses) $>$ (placebo item $\mathrm{CR}>$ item misses)] to specifically examine what distinguishes the groups for correct new decisions. Group differences in associative memory were evaluated by comparing the groups for fMRI activity in response to associative hits relative to associative misses [group $X$ association recall success interaction: haloperidol (associative hits $>$ associative misses) $>$ placebo (associative hits $>$ associative misses) and vice versa (Table 1)].

To assess group differences in functional connectivity between brain regions during associative retrieval, we conducted a psychophysiological interaction (PPI) analysis, as implemented in SPM12. To this end, we extracted the time course from left lateral PFC for each participant (peak voxel at $-34 / 38 /-8$, sphere radius of $8 \mathrm{~mm}$ ), which had been identified by the group $\times$ association recall success interaction analysis described at the end of the previous paragraph and computed the interaction between the left lateral PFC time course and the associative hits $>$ associative miss contrast. Because of the relatively low amount of associative hits trials, we additionally also computed the interaction between the left lateral PFC time course and the item hits $>$ item miss contrast. This contrast was selected assuming that participants would attempt associative retrieval only for item hits but not for item misses and thus differential coupling for these more abundant trials might likewise reveal prefrontal coupling driven by association retrieval processes. For each participant, two new first level GLMs were computed with regressors for the left lateral PFC time course and for the interaction between the left lateral PFC time course and the associative hits $>$ associative miss contrast and the interaction between the left lateral PFC time course and the item hits $>$ item miss contrast, respectively. Group differences for the interactions between left lateral PFC time course and associative memory were probed using two-sample $t$ tests on the second level.

Moreover, we computed brain-behavior correlations within each group between associative memory performance as measured by the CAM score (see above), and (1) with the individual left lateral PFC activity (identified by the group $\times$ association recall success interaction analysis described above) as well as (2) with the striatal-prefrontal PPI coupling parameter resulting from the above PPI analysis.

We examined effects of reinstatement of faces and scenes by grouping trial onsets in a third first-level model into associative face hits (item hit trials with correct face responses), associative face FAs (item hit trials with incorrect scene responses), associative scene hits (item hit trials with correct scene responses), associative scene FAs (item hit trials with incorrect face responses), associative misses (item hit trials with forgotten associations), item CRs (correct new item-response trials), item FAs (incorrect old item-response trials collapsed across all associative responses), item misses (incorrect new item-response trials), and missing response trials. The corresponding five betas-of-interest for the association reinstatement analysis (associative face hits, associative face FAs, associative scene hits, associative scene FAs, associative misses) were fed into a second-level ANOVA. Main task effects of reinstatement of face and scene associations were probed with linear contrasts summed across groups for associative face hits $>$ associative scene hits and associative scene hits $>$ associative face hits, respectively, and group differences were evaluated using the group $X$ association reinstatement interactions: haloperidol (associative face hits $>$ associative scene hits) $>$ (placebo (associative face hits $>$ associative scene hits) and vice versa (Table 1 ).

Given the strong a priori hypothesis of involvement of the striatum in dopaminergic signaling and the hippocampal role in memory processes as well as presence of (presynaptic and postsynaptic) hippocampal dopamine receptors (Gangarossa et al., 2012; Etter and Krezel, 2014; Rocchetti et al., 2015), we used a small volume familywise error correction (SVCFWE) at $p<0.05$ based on anatomical masks ( $50 \%$ probability map) of the hippocampus and the striatum. The anatomical masks were created by combining the individual left and right nucleus accumbens, putamen, and caudate nucleus as well as the left and right hippocampus masks from the Harvard-Oxford subcortical structural atlases (Desikan et al., 2006) as implemented in FSL (RRID:SCR_002823) into one striatum and one hippocampus mask, respectively. For all other reported findings, wholebrain FWE-correction at cluster level at $p<0.05$ (using a cluster-forming height threshold at voxel-level of $p<0.001$; Eklund et al., 2016) was applied.

\section{Results}

\section{Behavioral data}

Demographics

The haloperidol and the placebo group did not differ with regard to age, weight or sex (Table 2). There was no significant pattern of received and guessed substance (33\% of haloperidol participants and $19 \%$ of the placebo participants guessed that they had received haloperidol, $\left.\chi_{(1, \mathrm{~N}=53)}^{2}=0.16, p=0.69\right)$ and no difference with regard to the certainty of guess $\left(t_{(51)}=0.47, p=0.64\right)$. There were no significant group differences in systolic blood pressure, diastolic blood pressure, or pulse relative to baseline. There were no significant group differences in reported side effects or in reported subjective feelings across measurements relative to baseline (16-item VAS grouped into the dimensions "alertness", "calmness", and "contentedness"; Bond and Lader, 1974; Clos et al., 2019). As expected, working memory performance did not differ between groups at baseline, neither when comparing the 

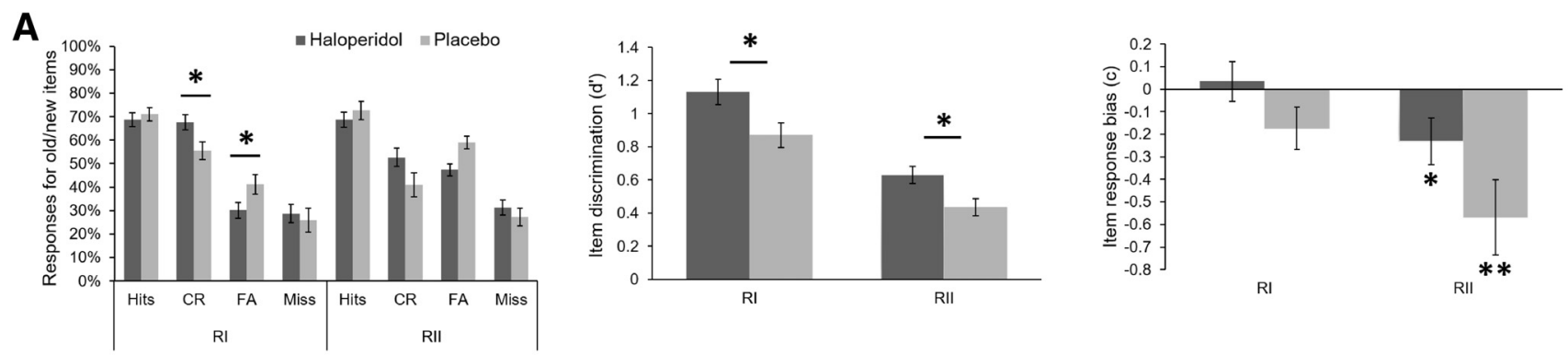

B

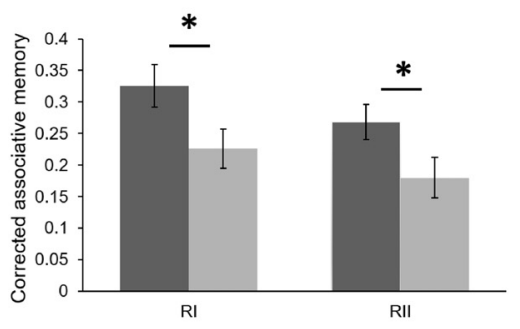

\section{C}

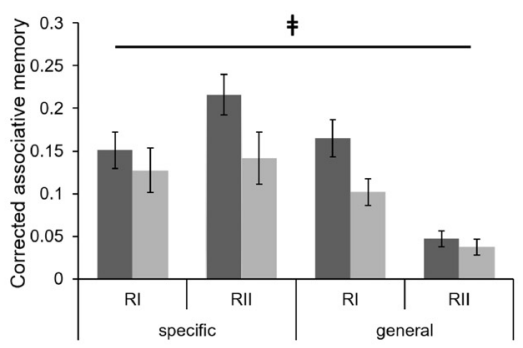

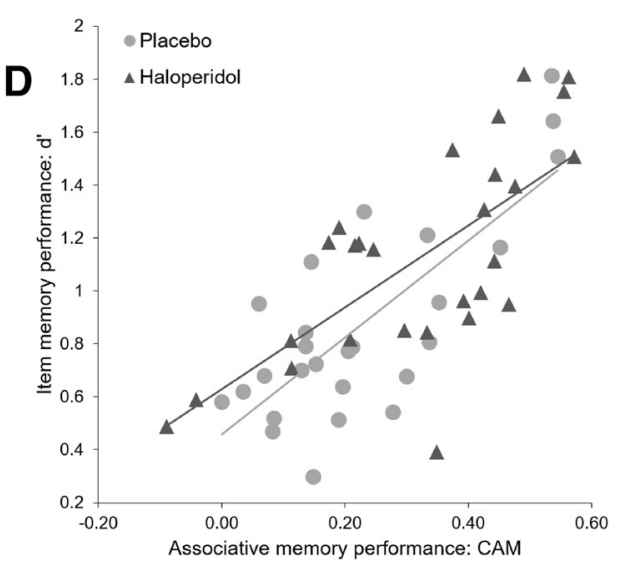

Figure 2. Behavioral effects in the haloperidol group (dark gray) and the placebo group (light gray) at Retrieval I (RI) under acute medication and at Retrieval II (RII). A, Item memory: frequencies of hit and CR responses (left), memory discrimination d' (middle), and response bias c (right). B, Associative memory for item hits: CAM overall (associative hits - associative FAs). C, Re-encoding effects on associative memory specificity: specific (left) and general (right) CAM. $\boldsymbol{D}$, Scatter plot showing the significant positive relationship between item memory and association memory performance per group at Retrieval I (triangle/circle markers). ${ }^{*} p<0.05,{ }^{* *} p<0.01$; $\ddagger$ significant group $\times$ time $\times$ specificity interaction at $p<0.05$. Error bars denote SEM.

tasks individually (all $p$ values $>0.11$ ), nor when summarizing them into a score based on the average standardized $z$-scores $\left(t_{(51)}=-0.87, p=0.39\right.$ ) or based on a PCA (first component; $\left.t_{(51)}=-0.85, p=0.40\right)$. In contrast to the previously observed impaired working memory under haloperidol in the full sample, post-drug working memory relative to baseline showed only trend-level significance for a reduction under haloperidol with the current sample (time $\times$ group interaction: $F_{(1,49)}=3.70, p=$ 0.060). See Clos et al. (2019) for more details. Moreover, the reaction time and the accuracy of categorization in the encoding task before drug administration were not different between groups (left/right arrow categorization: $t_{(50)}=0.25, p=0.80$, RT: $\left.t_{(50)}=0.32, p=0.98\right)$. Although the (nonsignificant) reduced working memory span under haloperidol together with the absence on drug effects on self-reported alertness are not indicative of attention-mediated drug effects on superior memory retrieval, future studies might consider to control more thoroughly for dopaminergic effects on attention using pre-/post-sustained attention tasks.

\section{Retrieval I}

Item memory

Independent samples $t$ tests revealed significantly higher discrimination performance $d^{\prime}$ for item memory (word recognition) in the haloperidol group under acute medication $\left[t_{(51)}=-2.47\right.$, $p=0.017$, Cohen's $d=0.68 /$ Pearson's $r=0.33$ (medium effect size)] but no difference in response bias c between groups $\left(t_{(51)}=\right.$ $-1.62, p=0.111$ ), indicating better item memory in the haloperidol group compared with the placebo group (Fig. 2A). Similar performance differences were obtained using the corrected hitrate (hit rate - FA rate), which was significantly increased from $30 \%$ (placebo group) to $39 \%$ [haloperidol group; $t_{(51)}=-2.46$, $p=0.017$, Cohen's $d=0.68$, Pearson's $r=0.33$ (medium effect size)]. Importantly, the relative frequencies of hit, CR, FA, and miss responses indicated that the enhanced discrimination performance was because of more correct rejections of new items in the haloperidol group (i.e., significantly more CRs and accordingly significantly less FAs; Fig. 2A).

The RT of the memory decision did not differ significantly between the groups across all trials (placebo: $1942 \mathrm{~ms}$, haloperidol: $1848 \mathrm{~ms}$; main effect of group: $\left.t_{(51)}=0.84, p=0.40\right)$. No significant group $\times$ condition interactions (with old/new rating or accuracy) for RT were observed (hits: placebo $2274 \mathrm{~ms}$, haloperidol: $2248 \mathrm{~ms}$; CRs: placebo $1689 \mathrm{~ms}$, haloperidol $1583 \mathrm{~ms}$; FAs: placebo $2297 \mathrm{~ms}$, haloperidol $2229 \mathrm{~ms}$; misses: placebo 1773 ms, haloperidol $1695 \mathrm{~ms}$; all $p>0.50$ ). However, it should be noted that the complex response requirements of the combined old-new and associative rating scale might confine the value of RT effects somewhat, as the multilevel decision is probably not captured well by a single response time.

\section{Association recall}

An independent samples $t$ test revealed that CAM for item hits (associative hit rate - associative FA rate) was significantly enhanced under acute haloperidol administration $\left[t_{(51)}=-2.18\right.$, $p=0.034$, Cohen's $d=0.60$, Pearson's $r=0.29$ (medium effect size)], indicating better associative memory in the haloperidol compared with the placebo group (Fig. $2 B$ ). Note that the CAM score can only be computed for item hit trials, because this is the only response category where associative hits may be present. Importantly, the improved association recall therefore cannot be explained merely by the better item memory performance reported above as the amount of item hits did not differ between groups (Fig. 2A). Both groups showed a similar significant posi- 
Table 3. Descriptive statistics of associative responses in item hit trials Retrieval $I^{a}$

\begin{tabular}{|c|c|c|c|c|c|}
\hline & $\begin{array}{l}\text { General } \\
\text { associative } \\
\text { hits, } \%^{b}\end{array}$ & $\begin{array}{l}\text { General } \\
\text { associative } \\
\text { FAs, } \%^{b}\end{array}$ & $\begin{array}{l}\text { Specific } \\
\text { associative } \\
\text { hits, } \%^{c}\end{array}$ & $\begin{array}{l}\text { Specific } \\
\text { associative } \\
\text { FAs, \% }{ }^{c}\end{array}$ & $\begin{array}{l}\text { Associative } \\
\text { miss, } \%^{d}\end{array}$ \\
\hline Placebo: scene trials & $25.33(2.7)$ & $18.17(3.0)$ & $12.05(2.5)$ & $3.89(0.9)$ & $40.56(4.5)$ \\
\hline Haloperidol: scene trials & $30.11(3.3)$ & $14.26(1.8)$ & $10.92(2.0)$ & $2.62(1.0)$ & $42.09(4.5)$ \\
\hline Placebo: face trials & $29.27(3.2)$ & $16.96(2.5)$ & $18.18(3.4)$ & $1.65(0.5)$ & $33.97(3.9)$ \\
\hline Haloperidol: face trials & $30.31(2.3)$ & $10.88(2.3)$ & $22.38(3.1)$ & $1.57(0.5)$ & $34.86(3.4)$ \\
\hline
\end{tabular}

Numbers in parentheses denote SE.

${ }^{a}$ Frequencies of associative hits and FAs differ between groups (significant group $\times$ accuracy interaction: $F_{(1,51)}=5.04, p=0.029$, partial $\eta^{2}=0.09$ ) similarly across trial type and specificity (group $\times$ accuracy $\times$ trial type interaction: $F_{(1,51)}=0.17, p=0.686$; group $\times$ accuracy $\times$ specificity interaction: $F_{(1,51)}=1.80$, $p=0.186$; group $\times$ accuracy $\times$ trial type $\times$ specificity interaction: $F_{(1,51)}=0.35, p=0.559$ ).

${ }^{b}$ Scene or face response.

'Specific scene or specific face response.

${ }^{d}$ Association forgotten response.

tive correlation between item memory performance $\left(d^{\prime}\right)$ and associative memory performance (CAM: haloperidol group: $r=$ 0.69, $p<0.001$; placebo group: $r=0.77, p<0.001$; Fig. $2 D)$. Additionally, a $2 \times 2 \times 2 \times 2$ repeated-measures ANOVA with the factors group, accuracy (associative hit/FA), specificity (general/specific associative response), and trial type (scene/face) of the relative frequencies of associative hits and associative FAs demonstrated that better associative memory was due to both more associative hits and less associative FAs similarly across scene and face trials and across general and specific associative responses [significant group $\times$ accuracy interaction: $F_{(1,51)}=$ 5.04, $p=0.029$, partial $\eta^{2}=0.09$ (medium effect size); Table 3]. The frequency of associative miss responses for item hits did not differ between groups (group $\times$ type interaction: $F_{(1,51)}=0.04$, $p=0.834$; main effect of group: $\left.F_{(1,51)}=0.05, p=0.831\right)$.

Association responses moreover existed for item FA trials (which, by definition, are always incorrect as no associations had been learned for new word items). To test for drug effects on the tendency to report associations, we compared the frequency of general versus specific associative responses between groups for item FA trials. A $2 \times 2$ repeated-measures ANOVA with the factors group and specificity of the relative frequencies of associative responses revealed no differences between groups (group $\times$ specificity interaction: $\left.F_{(1,51)}=1.96, p=0.660\right)$. Together, the associative response pattern for item FAs indicates that there were no group differences for associative responses for falsely remembered word items.

\section{Retrieval II}

Data from the second memory test are only available for 51 participants, as one haloperidol and one placebo participant did not return for the behavioral retrieval task $3 \mathrm{~d}$ later.

\section{Item memory}

Discrimination performance for item memory $\left(d^{\prime}\right)$ was significantly lower at Retrieval II for both groups compared with Retrieval I (main effect of time: $F_{(1,49)}=114.86, p<0.001$; group $\times$ time interaction: $\left.F_{(1,49)}=0.27, p=0.609\right)$ and overall significantly higher in the haloperidol group across retrieval time points [main effect of group: $F_{(1,49)}=7.09, p=0.010$, partial $\eta^{2}=0.13$ (medium effect size); Fig. 2A)]. The absence of a significant group $\times$ time interaction indicated that there was no dopaminergic effect on item memory retrieval performance through reencoding during the intermediary item recognition at Retrieval I, as the memory discrimination advantage under haloperidol remained constant across the first and the second retrieval time point. Both groups showed a significant bias toward "old"- decisions at Retrieval II (placebo group: $t_{(24)}=-3.41, p=0.002$; haloperidol group: $t_{(25)}=-2.22, p=0.036$ ), which was furthermore significantly greater than at Retrieval I (main effect of time: $F_{(1,49)}=14.18, p<0.001$; time $\times$ group interaction: $F_{(1,49)}=$ $0.69, p=0.410$; Fig. $2 A$ ). This strong old-bias at Retrieval II was probably at least in part due to the encoding of the lure word items at Retrieval I.

The RT of the memory decision did not differ significantly between the groups across all trials (placebo: $1139 \mathrm{~ms}$, haloperidol: 1106 ms; main effect of group: $\left.F_{(1,49)}=0.25, p=0.620\right)$ but showed a significant group $X$ accuracy rating interaction $\left[F_{(1,49)}=8.29, p=0.006\right.$, partial $\eta^{2}=0.15$ (medium effect size) , indicating that correct trials had similar response times in both groups, but the haloperidol group responded faster on incorrect trials at Retrieval II compared with the placebo group (hits: placebo 1337 ms, haloperidol: 1413 ms; CRs: placebo 994 ms, haloperidol $944 \mathrm{~ms}$; FAs: placebo $1327 \mathrm{~ms}$, haloperidol $1181 \mathrm{~ms}$; misses: placebo $1103 \mathrm{~ms}$, haloperidol $1007 \mathrm{~ms}$ ). However, it should be noted that the complex response requirements of the combined old-new and associative rating scale might confine the value of RT effects somewhat, as the multilevel decision is probably not captured well by a single response time.

\section{Association recall}

CAM was significantly lower at Retrieval II for both groups compared with Retrieval I (main effect of time: $F_{(1,49)}=14.36, p<$ 0.001 ) and overall significantly higher in the haloperidol group across retrieval time points [main effect of group: $F_{(1,49)}=4.20$, $p=0.046$, partial $\eta^{2}=0.08$ (medium effect size); group $\times$ time interaction: $F_{(1,49)}=0.15, p=0.903$; Fig. $2 B$ ]. The relative frequencies of associative hits and associative FAs at Retrieval II demonstrated that better associative memory was due to both more associative hits and less associative FAs particularly for face trials similarly across general, categorical and specific categorical associative responses [significant group $\times$ accuracy $\times$ trial type interaction: $F_{(1,49)}=4.19, p=0.046$, partial $\eta^{2}=0.08$ (medium effect size); Table 4].

Although the overall CAM score remained constantly enhanced under haloperidol across retrieval time points, analyzing the associative memory performance separately for general and specific CAM revealed a significant group $\times$ time $\times$ specificity interaction: $F_{(1,49)}=6.41, p=0.015$, partial $\eta^{2}=0.12$ (mediumlarge effect size). This result indicates that the specific (but not the general) CAM-score was increased from Retrieval I to Retrieval II in the haloperidol compared with the placebo group (Fig. 2C). In other words, the specificity of associative recall at Retrieval II was boosted by dopamine affecting re-encoding during and/or consolidation processes following Retrieval I. This effect was also reproduced in the analysis of associative response frequencies across retrieval time points, which likewise revealed this significant group $\times$ time $\times$ accuracy $\times$ specificity interaction: $F_{(1,49)}=$ 6.41, $p=0.015$, partial $\eta^{2}=0.12$ (medium-large effect size) in addition to a significant group $\times$ accuracy interaction: $F_{(1,49)}=$ 4.18, $p=0.046$ partial $\eta^{2}=.08$ (medium effect size), suggesting that although better associative memory was due to both more associative hits and less associative FAs similarly across scene and face trials and across retrieval time points, the haloperidol group showed an increase in specific associative hit responses at $\mathrm{Re}$ trieval II.

Frequencies of associative miss responses did not differ between groups at Retrieval II (group $\times$ type interaction: $F_{(1,49)}=$ $0.75, p=0.392$; main effect of group: $F_{(1,49)}=0.58, p=0.451$; 
Table 4. Descriptive statistics of associative responses in item hit trials Retrieval II ${ }^{a}$

\begin{tabular}{|c|c|c|c|c|c|c|c|}
\hline & $\begin{array}{l}\text { General } \\
\text { associative hits, } \%^{b}\end{array}$ & $\begin{array}{l}\text { General } \\
\text { associative FAs, } \%^{b}\end{array}$ & $\begin{array}{l}\text { Subcategorical } \\
\text { associative hits, \% }\end{array}$ & $\begin{array}{l}\text { Subcategorical } \\
\text { associative FAs, }{ }^{c}\end{array}$ & $\begin{array}{l}\text { Specific subcategorical } \\
\text { associative hits, } \%^{d}\end{array}$ & $\begin{array}{l}\text { Specific subcategorical } \\
\text { associative } F A s, \%^{d}\end{array}$ & $\begin{array}{l}\text { Associative } \\
\text { miss, } \%{ }^{e}\end{array}$ \\
\hline Placebo: scene trials & $12.68(1.9)$ & $11.58(1.4)$ & $8.33(1.6)$ & $5.03(1.2)$ & $7.24(2.0)$ & $0.67(0.2)$ & $54.47(4.4)$ \\
\hline Haloperidol: scene trials & $11.98(1.8)$ & $11.85(1.4)$ & $9.93(1.4)$ & $5.01(1.0)$ & $9.29(1.8)$ & $0.43(0.2)$ & $51.49(3.6)$ \\
\hline Placebo: face trials & $16.17(2.0)$ & $9.77(1.7)$ & $11.57(1.5)$ & $3.57(1.1)$ & $10.83(2.6)$ & $0.38(0.2)$ & $47.70(4.6)$ \\
\hline Haloperidol: face trials & $15.28(1.4)$ & $6.00(1.1)$ & $18.36(1.9)$ & $3.14(0.9)$ & $14.56(2.6)$ & $0.31(0.1)$ & $42.34(3.3)$ \\
\hline
\end{tabular}

Numbers in parentheses denote SE.

${ }^{a}$ Frequencies of associative hits and FAs differ between groups particularly for face trials (significant group $\times$ accuracy $\times$ trial type interaction: $\left.F_{(1,49)}=4.19, p=0.046\right)$ similarly across specificity levels (group $\times$ accuracy $\times$ trial type $\times$ specificity interaction: $F_{(2,48)}=0.34, p=0.711$; group $\times$ accuracy $\times$ specificity interaction: $F_{(2,48)}=2.04, p=0.142 ;$ group $\times$ accuracy interaction: $\left.F_{(1,49)}=3.91, p=0.54\right)$.

${ }^{b}$ Scene or face response.

'Natural/man-made scene or female/male face response.

${ }^{d}$ Specific natural/man-made scene or specific female/male face response.

${ }^{e}$ Association forgotten response.

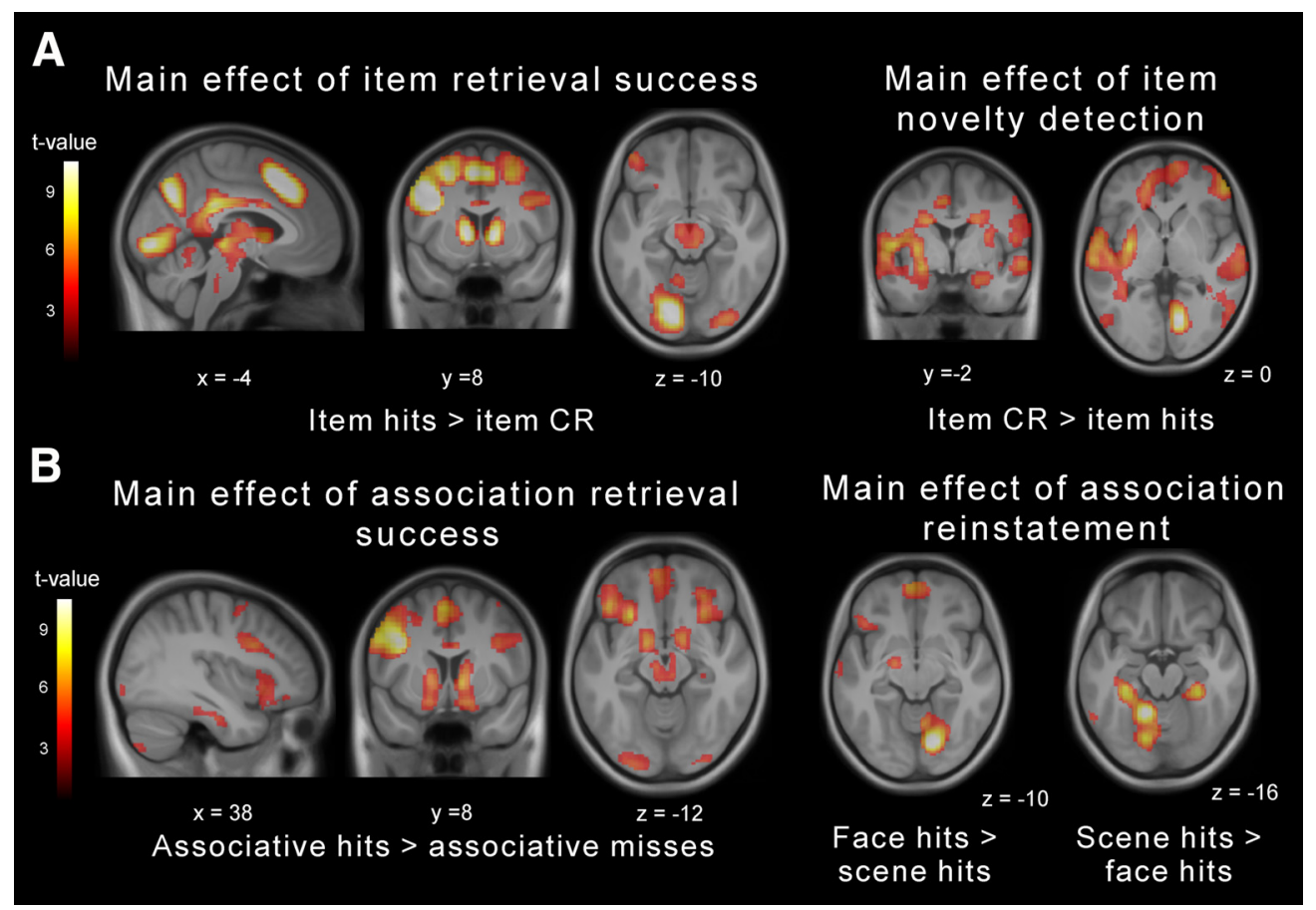

Figure 3. Main fMRI effects of retrieval across groups. $\boldsymbol{A}$, Item memory: retrieval success (left) and item novelty detection (right). $\boldsymbol{B}$, Associative memory: association retrieval success (left) and reinstatement of face and scene associations (right). All activation maps are thresholded at $p<0.05$ (FWE-corrected at cluster-level using a cluster forming threshold at voxel level of $p<0.001$ ).

Table 4) nor across retrieval time points (group $\times$ time interaction: $F_{(1,49)}=2.52, p=0.119$; group $\times$ time $\times$ type interaction: $\left.F_{(1,49)}=0.03, p=0.869\right)$. Association responses for item FAs did not differ in terms of specificity between groups at Retrieval II (group $\times$ specificity interaction: $\left.F_{(1,48)}=0.44, p=0.645\right)$ nor across retrieval time points (group $\times$ time interaction: $F_{(1,49)}=$ $0.37, p=0.547$; group $\times$ time $\times$ specificity interaction: $F_{(1,49)}=$ $0.10, p=0.754)$.

\section{fMRI results}

Main effects of item memory and association retrieval across groups

The main effect of item retrieval success (item hits $>$ item CRs summed across groups) revealed significant activation clusters in a network including the striatum, lateral PFC, substantia nigra (SN)/ventral tegmental area (VTA), anterior cingulate cortex (ACC), orbitofrontal cortex, insula, and precuneus. The inverse contrast of item novelty detection (item CRs $>$ item hits summed across groups) evoked significant activations in the bilateral amygdala extending into the hippocampus, as well as bilateral clusters in the superior temporal gyrus/inferior parietal lobe, medial PFC, visual cortex, and cerebellum and in the right ventrolateral PFC $(p<0.05$ whole-brain FWE-corrected; Fig. 3A).

The main effect of association retrieval success (associative hits $>$ associative misses summed across groups) revealed significant activity in a network including the bilateral inferior frontal gyrus (IFG), insula, ventromedial PFC, striatum, amygdala, SN/ VTA, thalamus, precuneus, posterior parietal cortex, visual cortex, left middle temporal gyrus (MTG), left inferior temporal gyrus (ITG)/fusiform/parahippocampal gyrus, and right fusiform /parahippocampal gyrus/hippocampus. Reinstatement effects of associative face retrieval (associative face hits $>$ associative scene hits summed across groups) was demonstrated in the right occipital fusiform gyrus, in the bilateral medial PFC, posterior parietal cortex, temporal pole, angular gyrus, and left IFG, whereas associative scene retrieval (associative scene hits $>$ associative face hits summed across groups) revealed significant activity in a network including the bilateral posterior parahippocampal gyrus, precuneus, and left posterior MTG/ITG $(p<$ 0.05 whole-brain FWE-corrected; Fig. 3B). 


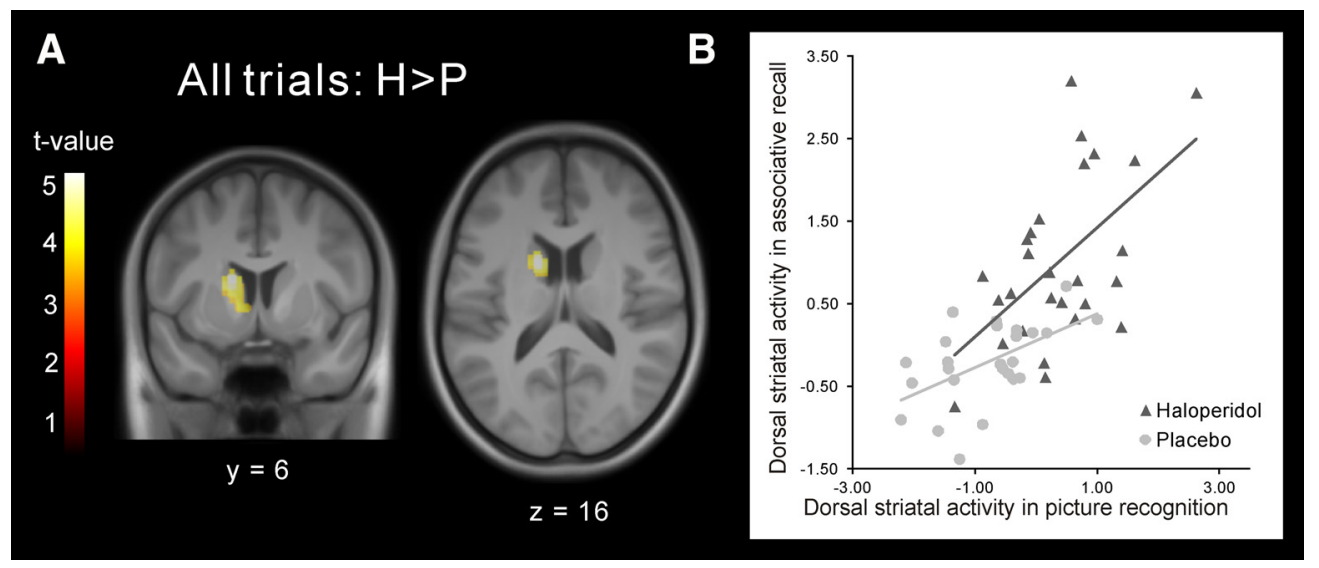

Figure 4. Main effect of haloperidol on $\mathrm{fMRl}$ activity. $A$, Haloperidol effect across all trials on brain activity compared with placebo. Note that increased activity in the right dorsal striatum was also present but its extent and height did not pass the FWE-corrected threshold of $p<0.05$. The inverse contrast (placebo $>$ haloperidol) revealed no significant activity. All activation maps are thresholded at $p<0.05$ (FWE-corrected at cluster-level using a cluster-forming threshold at voxel level of $p<0.001$ ). $\boldsymbol{B}$, Scatter plot showing the individual striatal activity across all trials in the current associative memory recall task and in the previous recognition memory task (Clos et al., 2019) in the haloperidol (dark gray) and the placebo groups (light gray).

\section{Main effect of haloperidol}

Group differences across all retrieval trials revealed significantly higher activation in the dorsal striatum in the haloperidol group compared with the placebo group in response to word onset (only the cluster in the left caudate nucleus was significant at $p<$ 0.05 when correcting for multiple comparisons; Fig. 4; Table 5). No other significant activity differences were observed between the groups in either direction across all trials. This selectively and specifically increased striatal activity in the haloperidol group was very similar to the increased striatal activity during picture recognition measured in this participant sample $1 \mathrm{~h}$ before (Clos et al., 2019), which was taken as evidence that the D2 antagonist primarily blocked the presynaptic D2-autoreceptors, resulting in increased DA-stimulation of the striatum. Indeed, the individual activity in the striatum peak voxel was significantly correlated across all trials of the associative and the recognition memory task in both groups (Fig. 4B), highlighting that the striatal signal was stable across time within participants.

\section{Effects of haloperidol on new item detection}

Based on the behavioral group differences in response to new items only (higher accuracy for new items under acute haloperidol), we analyzed the fMRI data for group differences with regard to new items. Using old items as a comparison, the haloperidol group showed significantly higher activity for factual new items in a network including bilateral temporoparietal regions (angular gyrus, superior and middle temporal gyrus), the bilateral dorsal and ventral striatum, ACC, the SN/VTA, left amygdala $(p<0.05$ whole-brain FWE-corrected) and the right hippocampus $(p<$ 0.05 FWE-SVC); Fig. 5A; Table 5). As visual inspection of the $\beta$ values suggested that the right hippocampal cluster was particularly driven by group differences in CR trials (Fig. $5 A, \beta$ plot), we quantified this using a minimum statistic conjunction of comparing groups for item CRs relative to item hits, item FAs, and item misses. Indeed, items CR trials differed between groups in the right hippocampus compared with all other trials (higher activity under haloperidol; $p<0.05$ FWE-SVC; Table 5). Note that although the $\beta$ plot in Figure $5 \mathrm{~A}$ suggests that the hippocampus in the placebo group does not respond to novelty, this effect is merely absent in this part of the hippocampus. In fact, the more anterior hippocampus showed bilaterally a novelty effect (i.e., a positive signal for item CRs and a deactivation for item hits) in both the haloperidol and in the placebo group (Fig. $3 A$, right).

Table 5. Peak activations group differences

\begin{tabular}{llllll}
\hline Region & $x$ & $y$ & $z$ & $z$-score & size \\
\hline
\end{tabular}

All trials: $H>P$

$\mathrm{L}$ caudate nucleus

Item memory: higher activity for factual new items for $H>P($ group $\times$ new/old $)$

R putamen

$R$ caudate nucleus

L nucleus accumbens

$R$ nucleus accumbens

L amygdala (CM; Amunts et al., 2005)

L putamen

LSN/VTA

$\mathrm{L}$ cerebellum

L fusiform gyrus (FG3; Lorenz et al., 2017)

R angular gyrus (PGa; Caspers et al., 2006)

$\mathrm{R}$ superior temporal gyrus

LACC

RACC

Langular gyrus

L superior temporal sulcus

R middle temporal gyrus

R hippocampus (subiculum (Amunts et al., 2005)

Item memory: higher activity for CR for

$H>P$ (group $\times$ CR conjunction)

R hippocampus (CA2; Amunts et al., 2005)

Associative memory: higher activity for associative hits for $H>P$ (group $\times$ association recall success)

L IFG p. Orbitalis

LMFG

R inferior parietal cortex

Psychophysiological interaction: increased coupling with L IFG for item hits > item miss for $H>P$

R putamen

$R$, Right; $L$, left; $H$, haloperidol; $P$, placebo.

$x-y-z$ Coordinates refer to the peak voxel in MNI space thresholded at $p<0.05$ (FWE-corrected at cluster-level; for hippocampus and striatum at small volume level where indicated). No significant activations were observed for the inverse contrasts.

${ }^{a}$ Small volume FWE-corrected using the bilateral anatomical hippocampus mask.

${ }^{b}$ Small volume FWE-corrected using the bilateral anatomical striatum mask.

Effects of haloperidol on association recall

To characterize the brain mechanisms reflecting the superior association recall performance under acute haloperidol, we compared correctly retrieved association trials (associative hits) with 


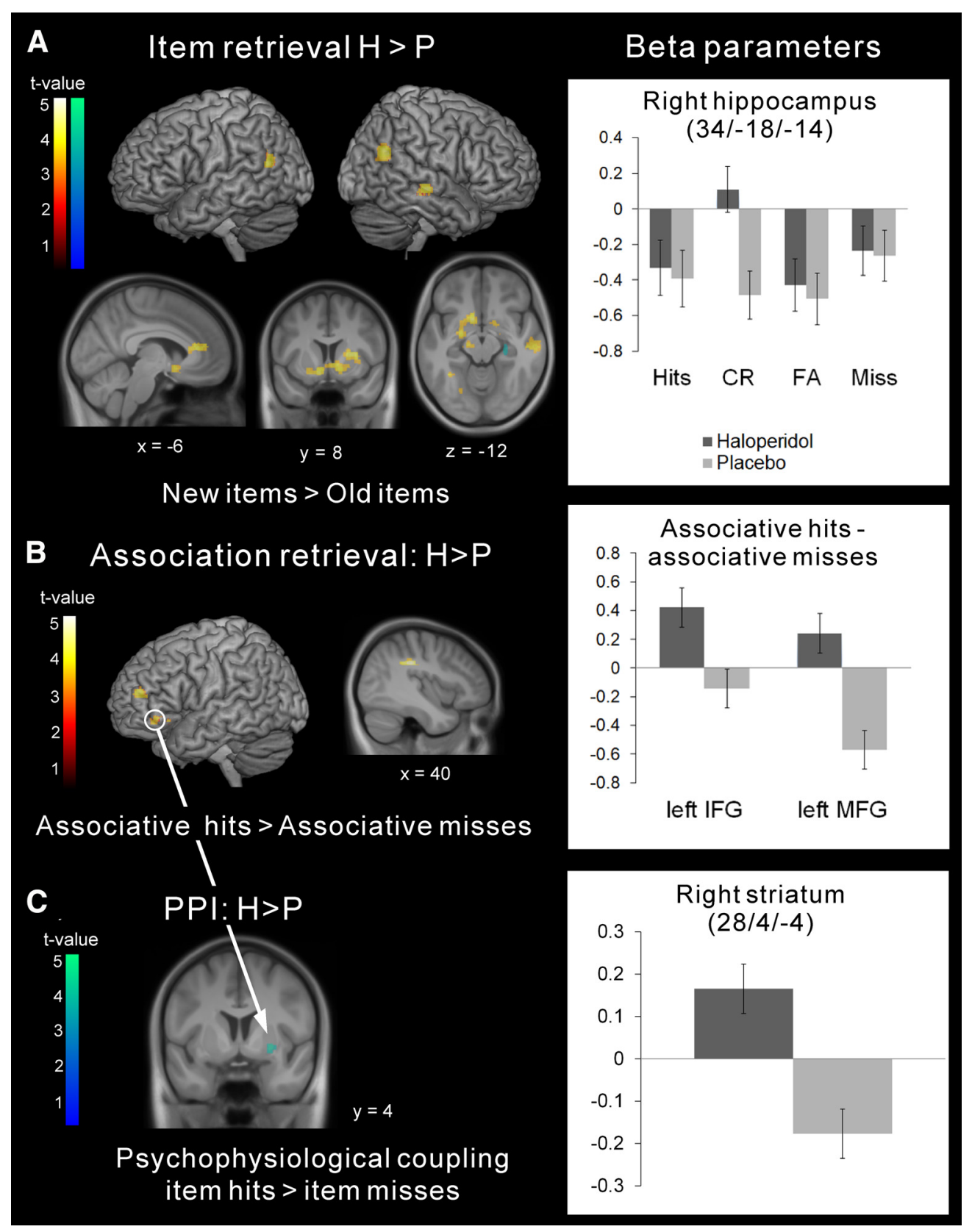

Figure 5. Effects of haloperidol on $\mathrm{fMRI}$ activity in memory retrieval. $A$, Item memory: higher activity for factual new items under haloperidol compared with placebo (haloperidol $[($ item $C R+$ item FA) $>$ (item hits + item misses)] $>$ placebo [(item CR + item FA) > (item hits + item misses)]). Right, Beta plot shows hippocampal activity across all item trials for the haloperidol group (dark gray) and the placebo group (light gray). $\boldsymbol{B}$, Association memory: higher activity for association retrieval success under haloperidol compared with placebo [haloperidol (associative hits $>$ associative misses) $>$ placebo (associative hits $>$ associative misses)]. Right, Beta plot shows differential prefrontal activity for associative hits $>$ associative misses between the haloperidol group (dark gray) and the placebo group (light gray). Note that we plotted the differential activity rather than individual betas because of the many regressors in the model. C, Increased coupling from the left lateral PFC to the right striatum for item hits $>$ item misses for haloperidol compared with placebo. Right, Beta plot shows differential prefrontal-striatal coupling parameter for item hits $>$ item misses between the haloperidol group (dark gray) and the placebo group (light gray). The inverse contrasts (placebo $>$ haloperidol) revealed no significant activity. All activation maps are thresholded at $p<0.05$ (warm colors: FWE-corrected at cluster-level using a cluster forming threshold at voxel level of $p<0.001$; cold colors: small volume FWE-corrected using anatomical masks).

forgotten association trials (associative misses) between groups. This group $\times$ association recall success interaction revealed significantly higher activation under haloperidol in the left lateral PFC (left middle frontal gyrus and left inferior frontal gyrus pars orbitalis) in addition to activity in the right inferior parietal lobe $(p<0.05$ whole-brain FWE-corrected) compared with placebo (Fig. 5B; Table 5). To examine the role of the PFC in associative retrieval in more detail, we conducted a PPI analysis seeded from the left lateral PFC to test for group differences in prefrontal connectivity during associative retrieval with other brain regions. Whereas the PPI for associative hits $>$ associative misses revealed no significant effects, prefrontal coupling for item hits $>$ item misses with the striatum was significantly higher in the haloperidol group compared with placebo (right putamen; $p<0.05$ FWE-SVC; Fig. 5C; Table 5). Assuming that participants would attempt associative retrieval only for item hits but not for item misses, this differential coupling suggests that increased prefrontal-striatal coupling contributed to the superior association 
retrieval performance under haloperidol. Of potential interest, also the prefrontal coupling to the hippocampus was enhanced for this contrast under haloperidol, albeit only at an uncorrected level (MNI $-26 /-24 /-18 ; p=0.26$ FWE-SVC, $z=2.81$ ). In contrast, individual brain-behavior correlations between associative memory performance (CAM) and the prefrontal-striatal coupling parameter from the above PPI as well the left lateral PFC activity for associative hits $>$ associative misses revealed neither significant correlations within the haloperidol group nor significant group differences for these correlations. Note that this absence of brain-behavior correlations in particular for left lateral PFC activity for associative hits $>$ associative misses might also be because of the low reliability of difference scores at the individual level (Infantolino et al., 2018), especially when the constituents are correlated (in our case the left lateral PFC signal for associative hits and associative misses were highly correlated in both groups (haloperidol group: $r=0.85, p<0.001$; placebo group: $r=0.83, p<0.001)$.

Finally, although there were clear reinstatement of associative face retrieval and associative scene retrieval across groups (Fig. $3 B$, right), we observed no significant group differences of face or scene reinstatement activity in these stimulusselective regions.

\section{Discussion}

In line with our previous results for picture recognition (Clos et al., 2019), memory accuracy in the association recall task was significantly enhanced under 2 mg of the D2 antagonist haloperidol administered during the retrieval phase. The similarity of increased striatal activity in the haloperidol group in the picture recognition task (Clos et al., 2019) and in the cued association recall task moreover suggests that haloperidol potentiated dopamine release from the SN/VTA by blocking the presynaptic autoreceptors (Pehek, 1999; Schwarz et al., 2004; Chen et al., 2005) also in the present cued association recall task.

Importantly, our current results extend the previously reported dopaminergic effects on item recognition enhancement (Clos et al., 2019) to the domain of association retrieval and deepen our understanding of the involved brain regions. Finally, this study also explored whether re-encoding of retrieved memories is affected by the dopaminergic modulation.

First, the behavioral results demonstrate that haloperidol improved association recall in addition to item recognition performance. This finding is remarkable because retrieval of associated information involves different mechanisms than item recognition. In particular, association memory relies more heavily on strategic search and top-down operations in the lateral PFC and the parietal cortex (Spaniol et al., 2009; Kim, 2013). In particular the left lateral PFC has been implicated in association retrieval for semantic material (Badre and Wagner, 2002, 2007). Indeed, our fMRI results for association recall highlight group differences in left lateral PFC and in right parietal lobe activity suggesting that improved prefrontal and parietal strategic search and monitoring processes contributed to the enhanced association retrieval accuracy under haloperidol. The PFC is densely innervated by midbrain dopaminergic neurons and increased dopaminergic stimulation in the PFC has been linked with higher signal-tonoise ratio of prefrontal representations (Miller, 2000; Puig et al., 2014; Vander Weele et al., 2018). Also the parietal cortex has been shown to contain dopaminergic receptors (Ito et al., 2008). Additionally, the PPI results demonstrate that increased prefrontalstriatal interactions are involved in the superior associative memory performance under haloperidol, which might be inter- preted as increased prefrontal control processes that affect striatal responses under haloperidol during associative retrieval.

So far, only few studies investigated dopaminergic effects on source or association memory retrieval. One study provided preliminary evidence of impaired source and item memory under decreased dopaminergic transmission in humans using a lowdosed dopaminergic agonist (Montoya et al., 2008). Another study (Drag et al., 2009) demonstrated worse source memory performance in patients with Parkinson's disease but no effect on item memory and no effect of medication status (on/off dopaminergic medication). Moreover, a study using dopamine transporter heterozygous knock-out mice with chronically insufficient dopamine reuptake activity indicated that dopamine imbalance leads to deficits in associative spatial memory recall when pattern completion is required to solve the task (Li et al., 2010). However, none of these studies specifically examined dopamine effects during the retrieval phase.

Second, the improved item recognition under haloperidol in the current association retrieval task points to specific dopamine effects on recognition of verbal items. Although item recognition was significantly improved for both old and new pictorial items in the previous task (Clos et al., 2019), higher accuracy in word recognition was solely due to better performance on new words trials in the present study. This apparent difference between verbal and pictorial item recognition might be because of the fact that all words (in contrast to the scene pictures used in the previous study) have been encountered previously in other contexts. Possibly, this higher degree of pre-experimental familiarity (Kim, 2013; Persson and Söderlund, 2015) increased the salience of new items and rendered their detection more critical for good task performance and therefore more susceptible to dopaminergic influence. In a similar vein, a previous study showed that the salience of old or new items and striatal recruitment depend on the task mode (encoding vs retrieval; cf. Herweg et al., 2018). Moreover, there is indeed evidence for specific dopamine effects on FA rates in settings facilitating FA responses (Allen et al., 2012; Andreou et al., 2015; Guarnieri et al., 2016, 2017; Doss et al., 2018).

Our fMRI results suggest that the improved new item detection under haloperidol was linked with increased activity in a network including dopaminergic (SN/VTA and striatum) as well as temporoparietal regions, amygdala, the ACC and in particular the hippocampus. The hippocampus, which contains D1 and presynaptic (regulatory) as well as postsynaptic D2-receptors (Gangarossa et al., 2012; Etter and Krezel, 2014; Rocchetti et al., 2015), is known to be important for novelty detection (Daselaar et al., 2006; Kafkas and Montaldi, 2014; Herweg et al., 2018) as this requires the comparison between stored hippocampal patterns and current inputs (Lisman and Otmakhova, 2001; Lisman and Grace, 2005; Kumaran and Maguire, 2007). This hippocampal comparator mechanism (Hasselmo et al., 1995; Lisman and Grace, 2005) might have been improved by dopaminergic modulation for example via an enhancement of the signal-to-noise ratio (Warren et al., 2016; Yousif et al., 2016; Vander Weele et al., 2018) of hippocampal memory representations (Clos et al., 2019). Additionally, the ACC, SN/VTA and amygdala are part of the salience detection network (Seeley et al., 2007) and increased activity in these regions might reflect increased saliency signals under haloperidol contributing to improved novelty detection by directing attention to new stimuli. Furthermore, the increased bilateral temporoparietal activity under haloperidol in response to new stimuli suggests dopaminergic effects on the ventral attention system which comprises temporoparietal regions centered 
around the temporoparietal junction and is associated with bottom-up attention (Corbetta and Shulman, 2002). According to the attention to memory (AtoM) model (Cabeza et al., 2008; Ciaramelli et al., 2008), the ventral parietal cortex contributes to memory retrieval by detecting behaviorally relevant and salient memory cues conveyed by MTL structures, including the certainty that a stimulus is new (Cabeza et al., 2008). Our results indicate that the attentional capture by new word stimuli in the temporoparietal regions detected in the MTL is facilitated under haloperidol.

However, because drug administration took place directly after encoding, it is possible that haloperidol improved the consolidation of the word-picture pairs rather than boosting their retrieval directly. In particular, synaptic consolidation, which depends on activation of D1-like receptors (Clopath, 2012), is instantiated in the first few hours post-learning and sets the stage for long-term memory. In a similar vein as reward during encoding was shown to affect later memory retrieval via reward effects on post-encoding rest and overnight consolidation (Braun et al., 2018), post-encoding dopaminergic activity elicited by haloperidol before the start of the cued associative recall task might have modified the salience linked with the word-picture associations. Yet, such dopaminergic consolidation effects cannot explain the improved recognition performance in our previous picture recognition paradigm (Clos et al., 2019) where the encoding took place $1 \mathrm{~d}$ before drug administration. Therefore, it seems rather unlikely that the current superior item and associative memory retrieval should entirely be due to dopaminergic effects on consolidation.

Finally, the increased specificity of associative retrieval in the haloperidol group from the first to the second retrieval suggests that dopamine affected re-encoding of associations through the intermediary memory test. This dopaminergic re-encoding effect indicates that dopaminergic signaling contributes to the strengthening of memory traces in a testing situation. For example, it has been proposed that striatal dopamine signals behavioral relevance and future utility of memories (Scimeca and Badre, 2012). More generally, this result is in accordance with previous studies reporting an increase in memory specificity for repeated testing of paired associates (Sommer et al., 2008) and striatal activation for the testing effect (Wing et al., 2013; van den Broek et al., 2013). Although re-encoding during the first retrieval appears to be a necessary prerequisite for the emergence of this effect here, it remains of course possible that dopaminergic processes during the consolidation of the retrieved content following the first retrieval also contribute to the associative specificity effect.

Together, these findings highlight the importance of dopaminergic processes in episodic retrieval. In particular, recall of associations profits from dopaminergic enhancement of prefrontal monitoring mechanisms. Recognition of verbal items is facilitated by dopaminergic signaling, as this improves novelty detection in the hippocampus possibly via an increased signal-to-noise ratio and higher activity in salience networks. Additionally, dopamine seems to increase the specificity of associative recall by affecting re-encoding of associative memories through an intermittent retrieval.

\section{Limitations}

We here assume that acute administration of $2 \mathrm{mg}$ of haloperidol increases dopamine. Importantly, this effect has been directly demonstrated only in animals and might depend on the specific experimental conditions in addition to the dose range determin- ing the amount of presynaptic versus postsynaptic blockade. Moreover, all dopamine studies face the challenge of the often heterogeneous and complex pharmacological actions and illunderstood regulatory mechanisms, which might not affect each participant to the same degree. Eventually, additional studies using multimodal methodological approaches and preferably within-subject crossover designs reducing interindividual variance are required to examine dopaminergic effects on memory retrieval in more detail.

\section{References}

Allen P, Chaddock CA, Howes OD, Egerton A, Seal ML, Fusar-Poli P, Valli I, Day F, McGuire PK (2012) Abnormal relationship between medial temporal lobe and subcortical dopamine function in people with an ultra high risk for psychosis. Schizophr Bull 38:1040-1049.

Amunts K, Kedo O, Kindler M, Pieperhoff P, Mohlberg H, Shah NJ, Habel U, Schneider F, Zilles K (2005) Cytoarchitectonic mapping of the human amygdala, hippocampal region and entorhinal cortex: intersubject variability and probability maps. Anat Embryol 210:343-352.

Andreou C, Bozikas VP, Luedtke T, Moritz S (2015) Associations between visual perception accuracy and confidence in a dopaminergic manipulation study. Front Psychol 6:414.

Badre D, Wagner AD (2002) Semantic retrieval, mnemonic control, and prefrontal cortex. Behav Cogn Neurosci Rev 1:206-218.

Badre D, Wagner AD (2007) Left ventrolateral prefrontal cortex and the cognitive control of memory. Neuropsychologia 45:2883-2901.

Bond A, Lader M (1974) The use of analogue scales in rating subjective feelings. Br J Med Psychol 47:211-218.

Braun EK, Wimmer GE, Shohamy D (2018) Retroactive and graded prioritization of memory by reward. Nat Commun 9:4886.

Cabeza R, Ciaramelli E, Olson IR, Moscovitch M (2008) The parietal cortex and episodic memory: an attentional account. Nat Rev Neurosci 9:613625.

Caspers S, Geyer S, Schleicher A, Mohlberg H, Amunts K, Zilles K (2006) The human inferior parietal cortex: cytoarchitectonic parcellation and interindividual variability. Neuroimage 33:430-448.

Chen YC, Choi JK, Andersen SL, Rosen BR, Jenkins BG (2005) Mapping dopamine D2/D3 receptor function using pharmacological magnetic resonance imaging. Psychopharmacology 180:705-715.

Chen YC, Galpern WR, Brownell AL, Matthews RT, Bogdanov M, Isacson O, Keltner JR, Beal MF, Rosen BR, Jenkins BG (1997) Detection of dopaminergic neurotransmitter activity using pharmacologic MRI: correlation with PET, microdialysis, and behavioral data. Magn Reson Med 38: 389-398

Choi JK, Chen YI, Hamel E, Jenkins BG (2006) Brain hemodynamic changes mediated by dopamine receptors: role of the cerebral microvasculature in dopamine-mediated neurovascular coupling. Neuroimage 30: $700-712$.

Chugh Y, Saha N, Sankaranarayanan A, Sharma PL (1991) Possible mechanism of haloperidol-induced enhancement of memory retrieval. Methods Find Exp Clin Pharmacol 13:161-164.

Ciaramelli E, Grady CL, Moscovitch M (2008) Top-down and bottom-up attention to memory: a hypothesis (AtoM) on the role of the posterior parietal cortex in memory retrieval. Neuropsychologia 46:1828-1851.

Clopath C (2012) Synaptic consolidation: an approach to long-term learning. Cogn Neurodyn 6:251-257.

Clos M, Schwarze U, Gluth S, Bunzeck N, Sommer T (2015) Goal- and retrieval-dependent activity in the striatum during memory recognition. Neuropsychologia 72:1-11.

Clos M, Sommer T, Schneider SL, Rose M (2018) Enhanced transformation of incidentally learned knowledge into explicit memory by dopaminergic modulation. PLoS One 13:e199013.

Clos M, Bunzeck N, Sommer T (2019) Dopamine is a double-edged sword: dopaminergic modulation enhances memory retrieval performance but impairs metacognition. Neuropsychopharmacology 44:555-563.

Cools R (2016) The costs and benefits of brain dopamine for cognitive control. Wiley Interdiscip Rev Cogn Sci 7:317-329.

Corbetta M, Shulman GL (2002) Control of goal-directed and stimulusdriven attention in the brain. Nat Rev Neurosci 3:201-215.

Daselaar SM, Fleck MS, Cabeza R (2006) Triple dissociation in the medial 
temporal lobes: recollection, familiarity, and novelty. J Neurophysiol 96:1902-1911.

Desikan RS, Ségonne F, Fischl B, Quinn BT, Dickerson BC, Blacker D, Buckner RL, Dale AM, Maguire RP, Hyman BT, Albert MS, Killiany RJ (2006) An automated labeling system for subdividing the human cerebral cortex on MRI scans into gyral based regions of interest. Neuroimage 31: 968-980.

Dobbins IG, Han S (2006) Cue- versus probe-dependent prefrontal cortex activity during contextual remembering. J Cogn Neurosci 18:1439-1452.

Doss MK, Weafer J, Gallo DA, de Wit H (2018) $\Delta$ 9-Tetrahydrocannabinol at retrieval drives false recollection of neutral and emotional memories. Biol Psychiatry 84:743-750.

Drag LL, Bieliauskas LA, Kaszniak AW, Bohnen NI, Glisky EL (2009) Source memory and frontal functioning in Parkinson's disease. J Int Neuropsychol Soc 15:399-406.

Dugast C, Brun P, Sotty F, Renaud B, Suaud-Chagny MF (1997) On the involvement of a tonic dopamine D2-autoinhibition in the regulation of pulse-to-pulse-evoked dopamine release in the rat striatum in vivo. Naunyn Schmiedebergs Arch Pharmacol 355:716-719.

Eklund A, Nichols TE, Knutsson H (2016) Cluster failure: why fMRI inferences for spatial extent have inflated false-positive rates. Proc Natl Acad Sci U S A 113:7900-7905.

Etter G, Krezel W (2014) Dopamine D2 receptor controls hilar mossy cells excitability. Hippocampus 24:725-732.

Feinberg DA, Moeller S, Smith SM, Auerbach E, Ramanna S, Gunther M, Glasser MF, Miller KL, Ugurbil K, Yacoub E (2010) Multiplexed echo planar imaging for sub-second whole brain FMRI and fast diffusion imaging. PLoS One 5:e15710.

Ford CP (2014) The role of D2-autoreceptors in regulating dopamine neuron activity and transmission. Neuroscience 282:13-22.

Frank MJ, O’Reilly RC (2006) A mechanistic account of striatal dopamine function in human cognition: psychopharmacological studies with cabergoline and haloperidol. Behav Neurosci 120:497-517.

Gangarossa G, Longueville S, De Bundel D, Perroy J, Hervé D, Girault JA, Valjent E (2012) Characterization of dopamine D1 and D2 receptorexpressing neurons in the mouse hippocampus. Hippocampus 22: 2199-2207.

Garris PA, Budygin EA, Phillips PE, Venton BJ, Robinson DL, Bergstrom BP, Rebec GV, Wightman RM (2003) A role for presynaptic mechanisms in the actions of nomifensine and haloperidol. Neuroscience 118:819-829.

Goldhahn D, Eckart T, Quasthoff U (2012) Building large monolingual dictionaries at the Leipzig corpora collection: from 100 to 200 languages. Proceedings of the Eighth International Conference on Language Resources and Evaluation, Istanbul, Turkey, March.

Griswold MA, Jakob PM, Heidemann RM, Nittka M, Jellus V, Wang J, Kiefer B, Haase A (2002) Generalized autocalibrating partially parallel acquisitions (GRAPPA). Magn Reson Med 47:1202-1210.

Guarnieri RV, Ribeiro RL, de Souza AA, Galduróz JC, Covolan L, Bueno OFA (2016) Effects of sulpiride on true and false memories of thematically related pictures and associated words in healthy volunteers. Front Psychiatry $7: 28$.

Guarnieri RV, Buratto LG, Gomes CFA, Ribeiro RL, de Souza AAL, Stein LM, GaldurózJC, Bueno OFA (2017) Haloperidol increases false recognition memory of thematically related pictures in healthy volunteers. Hum Psychopharmacol 32:e2563.

Han S, Huettel SA, Raposo A, Adcock RA, Dobbins IG (2010) Functional significance of striatal responses during episodic decisions: recovery or goal attainment? J Neurosci 30:4767-4775.

Hasegawa I, Fukushima T, Ihara T, Miyashita Y (1998) Callosal window between prefrontal cortices: cognitive interaction to retrieve long-term memory. Science 281:814-818.

Hasselmo ME, Schnell E, Barkai E (1995) Dynamics of learning and recall at excitatory recurrent synapses and cholinergic modulation in rat hippocampal region CA3. J Neurosci 15:5249-5262.

Herweg NA, Sommer T, Bunzeck N (2018) Retrieval demands adaptively change striatal Old/New signals and boost subsequent long-term memory. J Neurosci 38:745-754.

Infantolino ZP, Luking KR, Sauder CL, Curtin JJ, Hajcak G (2018) Robust is not necessarily reliable: from within-subjects fMRI contrasts to betweensubjects comparisons. Neuroimage 173:146-152.

Ito H, Takahashi H, Arakawa R, Takano H, Suhara T (2008) Normal data- base of dopaminergic neurotransmission system in human brain measured by positron emission tomography. Neuroimage 39:555-565.

Jaworski JN, Gonzales RA, Randall PK (2001) Effect of dopamine D2/D3 receptor antagonist sulpiride on amphetamine-induced changes in striatal extracellular dopamine. Eur J Pharmacol 418:201-206.

Kafkas A, Montaldi D (2014) Two separate, but interacting, neural systems for familiarity and novelty detection: a dual-route mechanism. Hippocampus 24:516-527.

Kessels RP, van den Berg E, Ruis C, Brands AM (2008) The backward span of the corsi block-tapping task and its association with the WAIS-III digit span. Assessment 15:426-434.

Kim H (2013) Differential neural activity in the recognition of old versus new events: an activation likelihood estimation meta-analysis. Hum Brain Mapp 34:814-836.

Knutson B, Gibbs SE (2007) Linking nucleus accumbens dopamine and blood oxygenation. Psychopharmacology 191:813-822.

Kumaran D, Maguire EA (2007) Which computational mechanisms operate in the hippocampus during novelty detection? Hippocampus 17:735-748

Li F, Wang LP, Shen X, Tsien JZ (2010) Balanced dopamine is critical for pattern completion during associative memory recall. PLoS One 5: e15401.

Lisman JE, Grace AA (2005) The hippocampal-VTA loop: controlling the entry of information into long-term memory. Neuron 46:703-713.

Lisman JE, Otmakhova NA (2001) Storage, recall, and novelty detection of sequences by the hippocampus: elaborating on the SOCRATIC model to account for normal and aberrant effects of dopamine. Hippocampus 11: 551-568.

Liu XL, Liang P, Li K, Reder LM (2014) Uncovering the neural mechanisms underlying learning from tests. PLoS One 9:e92025.

Lorenz S, Weiner KS, Caspers J, Mohlberg H, Schleicher A, Bludau S, Eickhoff SB, Grill-Spector K, Zilles K, Amunts K (2017) Two new cytoarchitectonic areas on the human mid-fusiform gyrus. Cereb Cortex 27:373-385.

Macmillan NA, Creelman CD (2004) Detection theory: a user's guide. New York: Psychology.

Miller EK (2000) The prefrontal cortex and cognitive control. Nat Rev Neurosci 1:59-65.

Miyashita Y, Hayashi T (2000) Neural representation of visual objects: encoding and top-down activation. Curr Opin Neurobiol 10:187-194.

Moeller S, Yacoub E, Olman CA, Auerbach E, Strupp J, Harel N, Uğurbil K (2010) Multiband multislice GE-EPI at 7 tesla, with 16 -fold acceleration using partial parallel imaging with application to high spatial and temporal whole-brain fMRI. Magn Reson Med 63:1144-1153.

Montoya A, Lal S, Menear M, Duplessis E, Thavundayil J, Schmitz N, Lepage M (2008) Apomorphine effects on episodic memory in young healthy volunteers. Neuropsychologia 46:292-300.

Pehek EA (1999) Comparison of effects of haloperidol administration on amphetamine-stimulated dopamine release in the rat medial prefrontal cortex and dorsal striatum. J Pharmacol Exp Ther 289:14-23.

Persson J, Söderlund H (2015) Hippocampal hemispheric and long-axis differentiation of stimulus content during episodic memory encoding and retrieval: an activation likelihood estimation meta-analysis. Hippocampus 25:1614-1631.

Puig MV, Antzoulatos EG, Miller EK (2014) Prefrontal dopamine in associative learning and memory. Neuroscience 282:217-229.

Rocchetti J, Isingrini E, Dal Bo G, Sagheby S, Menegaux A, Tronche F, Levesque D, Moquin L, Gratton A, Wong TP, Rubinstein M, Giros B (2015) Presynaptic D2 dopamine receptors control long-term depression expression and memory processes in the temporal hippocampus. Biol Psychiatry 77:513-525.

Roediger HL 3rd, Butler AC (2011) The critical role of retrieval practice in long-term retention. Trends Cogn Sci 15:20-27.

Rowland CA (2014) The effect of testing versus restudy on retention: a meta-analytic review of the testing effect. Psychol Bull 140:1432-1463.

Sara SJ (1986) Haloperidol facilitates memory retrieval in the rat. Psychopharmacology 89:307-310.

Schwarz A, Gozzi A, Reese T, Bertani S, Crestan V, Hagan J, Heidbreder C, Bifone A (2004) Selective dopamine $\mathrm{D}_{3}$ receptor antagonist SB277011-A potentiates phMRI response to acute amphetamine challenge in the rat brain. Synapse 54:1-10.

Schwarze U, Bingel U, Badre D, Sommer T (2013) Ventral striatal activity 
correlates with memory confidence for old- and new-responses in a difficult recognition test. PLoS One 8:e54324.

Scimeca JM, Badre D (2012) Striatal contributions to declarative memory retrieval. Neuron 75:380-392.

Seeley WW, Menon V, Schatzberg AF, Keller J, Glover GH, Kenna H, Reiss AL, Greicius MD (2007) Dissociable intrinsic connectivity networks for salience processing and executive control. J Neurosci 27:2349-2356.

Setsompop K, Gagoski BA, Polimeni JR, Witzel T, Wedeen VJ, Wald LL (2012) Blipped-controlled aliasing in parallel imaging for simultaneous multislice echo planar imaging with reduced g-factor penalty. Magn Reson Med 67:1210-1224.

Simons JS, Spiers HJ (2003) Prefrontal and medial temporal lobe interactions in long-term memory. Nat Rev Neurosci 4:637-648.

Sommer T, Schoell E, Büchel C (2008) Associative symmetry of the memory for object-location associations as revealed by the testing effect. Acta Psychol 128:238-248.

Spaniol J, Davidson PS, Kim AS, Han H, Moscovitch M, Grady CL (2009) Event-related fMRI studies of episodic encoding and retrieval: metaanalyses using activation likelihood estimation. Neuropsychologia 47:1765-1779.

Tomita H, Ohbayashi M, Nakahara K, Hasegawa I, Miyashita Y (1999) Topdown signal from prefrontal cortex in executive control of memory retrieval. Nature 401:699-703.

Unsworth N, Redick TS, Heitz RP, Broadway JM, Engle RW (2009) Complex working memory span tasks and higher-order cognition: a latent- variable analysis of the relationship between processing and storage. Memory 17:635-654.

van den Broek GS, Takashima A, Segers E, Fernández G, Verhoeven L (2013) Neural correlates of testing effects in vocabulary learning. Neuroimage 78:94-102.

Vander Weele CM, Siciliano CA, Matthews GA, Namburi P, Izadmehr EM, Espinel IC, Nieh EH, Schut EHS, Padilla-Coreano N, Burgos-Robles A, Chang CJ, Kimchi EY, Beyeler A, Wichmann R, Wildes CP, Tye KM (2018) Dopamine enhances signal-to-noise ratio in cortical-brainstem encoding of aversive stimuli. Nature 563:397-401.

Warren CM, Eldar E, van den Brink RL, Tona KD, van der Wee NJ, Giltay EJ, van Noorden MS, Bosch JA, Wilson RC, Cohen JD, Nieuwenhuis S (2016) Catecholamine-mediated increases in gain enhance the precision of cortical representations. J Neurosci 36:5699-5708.

Wiklund-Hörnqvist C, Andersson M, Jonsson B, Nyberg L (2017) Neural activations associated with feedback and retrieval success. NPJ Sci Learn 2:12.

Wing EA, Marsh EJ, Cabeza R (2013) Neural correlates of retrieval-based memory enhancement: an fMRI study of the testing effect. Neuropsychologia 51:2360-2370.

Xu J, Moeller S, Auerbach EJ, Strupp J, Smith SM, Feinberg DA, Yacoub E, Uğurbil K (2013) Evaluation of slice accelerations using multiband echo planar imaging at 3 T. Neuroimage 83:991-1001.

Yousif N, Fu RZ, Abou-El-Ela Bourquin B, Bhrugubanda V, Schultz SR, Seemungal BM (2016) Dopamine activation preserves visual motion perception despite noise interference of human V5/MT. J Neurosci 36: 9303-9312. 